\title{
Process Validation\& Contents Uniformity in Tablets via Quality Tools and Process Capabilities
}

\author{
SohailAnwer ${ }^{1}$, Kamran Ahmed ${ }^{2}$, Huma Nargis ${ }^{3}$, Afshan Bhatti ${ }^{4}$, \\ Waqar Hussain ${ }^{5}$ \\ Assistant Professor Department of Pharmaceutics. Faculty of Pharmacy.HamdardUniversity.Karachi. Pakistan \\ Assistant Professor Department of Pharmaceutics. Faculty of Pharmacy.HamdardUniversity.Karachi. Pakistan \\ PIQC Institute of Quality .HamdardUniversity.Karachi. Pakistan \\ PIQC Institute of Quality .HamdardUniversity.Karachi. Pakistan \\ PIQC Institute of Quality .HamdardUniversity.Karachi. Pakistan
}

\begin{abstract}
This study based on such concepts and provides an overview of the quality tools such as cause \& effect analysis, process capabilities and other quality tools like normality plots histograms. Box plots and other statistical techniques and a step-by-step approach for determining process capability. Which ultimately leads to overall process improvement and validation of that tablets. Which were manufactured at industrial lab. Faculty of pharmacy Hamdard university

The above approach is illustrated in present study. As part of a process validation, A $5 \mathrm{mg}$ tablet was evaluated for content and dosage uniformity. The specifications were $85-115 \%$. Three batches were made and sampled. Thirty tablets were taken throughout the production run for each batch and thus represented the entire batch. The sample size is selected to conform to USP chapter 〈905〉, Uniformity of dosage unit

The overall results in different interpretation were satisfactory as per normality plots histograms. Box plots and other statistical techniques . Furthermore over all process capability Cpk was found with 1.206. Which is acceptable indicator of process capability
\end{abstract}

Keywords: Process validation, Quality tools, Statistical techniques, Content Uniformity, Process capabilities

\section{Introduction}

The Food and Drug Administration has defined validation as "establishing documented evidence which provides a high degree of assurance that a specific process will consistently producing a product meeting its predetermined specifications and quality attributes. The requirement of a high degree of assurance raises the question, what is an appropriate and acceptable degree of assurance? We assert that three validation successive manufactured batches, all of whose samples meet specifications, are insufficient to provide a high degree of assurance.

A capability study measures the natural variability of a process. This variability is examined and compared to the specifications that must be met. The process is capable if the variability is consistent and small enough so that most of the dosage units produced will meet their specifications.

Kieffer $^{1}$ has maintained that the well-established statistical measures for process capability are excellent for qualifying the degree of assurance. He has further proposed that the acceptable degree of assurance should be set relative to the risk vs. benefit for the measured quality characteristic for the end user

\section{Plan of work}

i. Manufacturing of tablets is carried out at industrial lab. Faculty of pharmacy as per existing facility

ii. Evaluate each cause and their contributing leading to effect ( content and dosage uniformity) by cause and effect diagram proposed model of cause and effect diagram expressed in Fig $1^{2}$, while typical process flow of process mentioned in Fig 2 
Fig 1. Cause \& Effect Diagram Of Tablets Validation

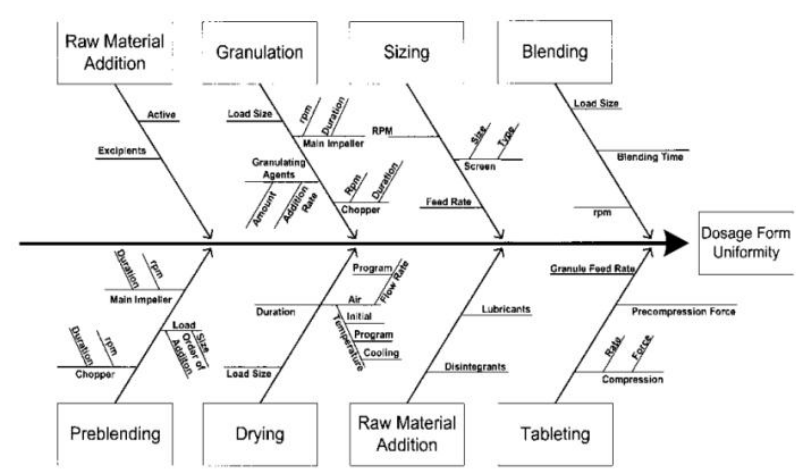

Fig. 2 PROCES FLOW TABLETS

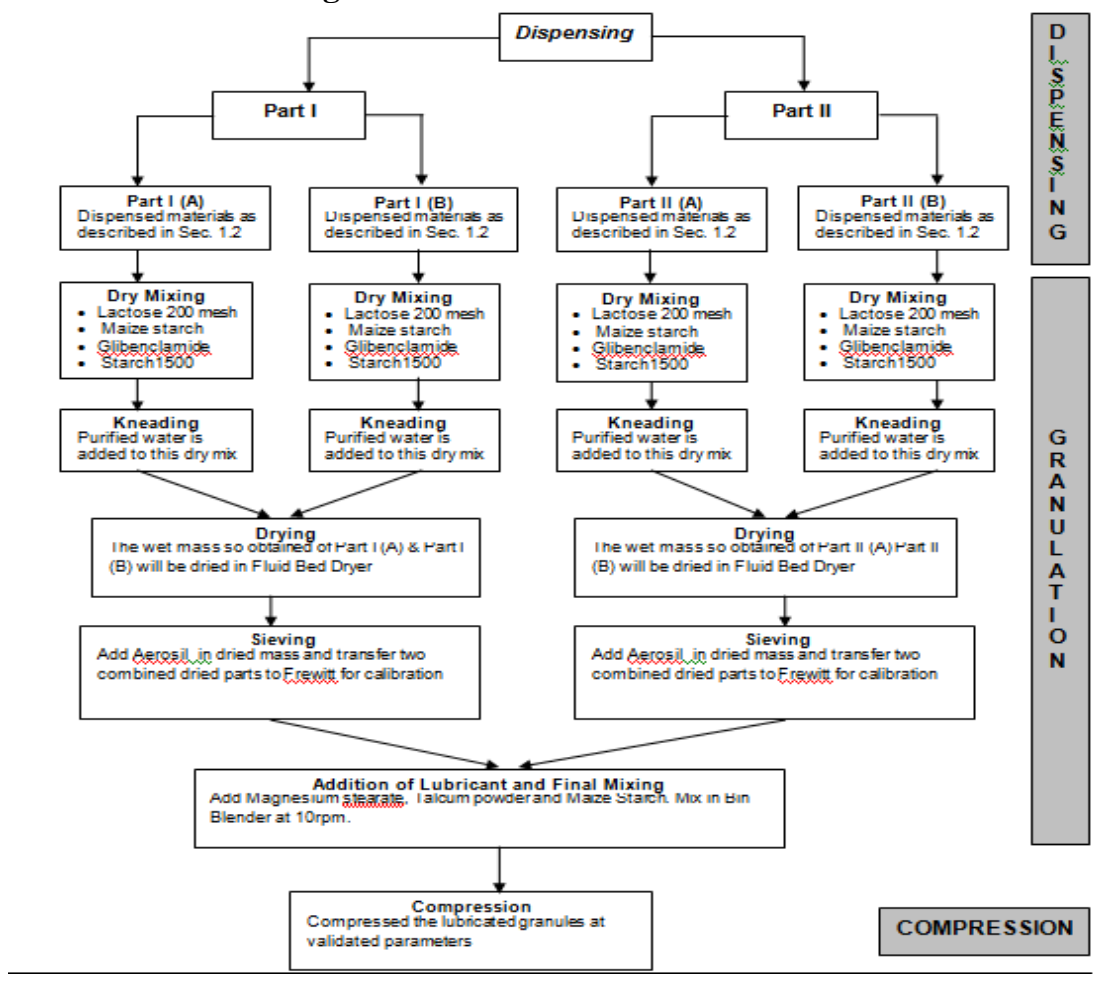

iii. Identify critical steps during manufacturing of tablets by control charts and other quality tools in order to facilitate process validation leading to content and dosage uniformity

iv. Content uniformity of $5 \mathrm{mg}$ tablets of Glibenclamide of three successive batches FI .F2 and F3 analyzed on spectrophotometer and then analyzed \% content \% of 30 tablets and tabulated in Table 1 .After each batch evaluated statistically A comparison is made of all of the batches . 
Table 1 Content Uniformity profile of Batch F 1, F 2 and F3

\begin{tabular}{|c|c|c|c|}
\hline TABLET & $\mathrm{F} 1$ & F2 & F3 \\
\hline 1 & 100.1 & 99.8 & 96.7 \\
\hline 2 & 97.6 & 106.3 & 98.5 \\
\hline 3 & 94.2 & 100 & 100.1 \\
\hline 4 & 99.5 & 99 & 106.9 \\
\hline 5 & 100.9 & 102.1 & 102 \\
\hline 6 & 105.4 & 104 & 104.1 \\
\hline 7 & 104.6 & 103.4 & 104.1 \\
\hline 8 & 99.2 & 97.7 & 95 \\
\hline 9 & 101.3 & 94.7 & 104.7 \\
\hline 10 & 100.0 & 99 & 105.2 \\
\hline 11 & 100.3 & 103.2 & 107.8 \\
\hline 12 & 105.9 & 104.3 & 102.6 \\
\hline 13 & 109.2 & 109.6 & 105.5 \\
\hline 14 & 96.9 & 98.1 & 104.1 \\
\hline 15 & 99.8 & 95.7 & 101.5 \\
\hline 16 & 100.1 & 105.6 & 103.4 \\
\hline 17 & 106.1 & 95 & 101.7 \\
\hline 18 & 99.1 & 101.9 & 100.5 \\
\hline 19 & 98.2 & 103.2 & 108.3 \\
\hline 20 & 96.8 & 104.3 & 112.9 \\
\hline 21 & 99.7 & 97.6 & 108 \\
\hline 22 & 104.8 & 96.1 & 102.9 \\
\hline 23 & 102.0 & 104.3 & 102. \\
\hline 24 & 101.7 & 103.1 & 99.8 \\
\hline 25 & 95.2 & 103.4 & 98.7 \\
\hline 26 & 98.3 & 93 & 96.2 \\
\hline 27 & 104.0 & 98.5 & 105.1 \\
\hline 28 & 102.8 & 99.9 & 108.1 \\
\hline 29 & 100.00 & 95.9 & 103.9 \\
\hline 30 & 99.4 & 103.1 & 101.2 \\
\hline
\end{tabular}

III. Application of Quality tools and Statistical Evaluation that

The summary of the three validation batches regarding content uniformity is shown in Table 1 Note

Data of all three batches is précised and can be explain by various statistical tools and techniques

\subsection{Normality Plots}

The data for batch F1,F2 and F3 first inspected visually with normality plots mentioned in Fig 3 ,

Fig 4 and Fig. 5 along with their statistical inferences in Table 1, 2 and 3

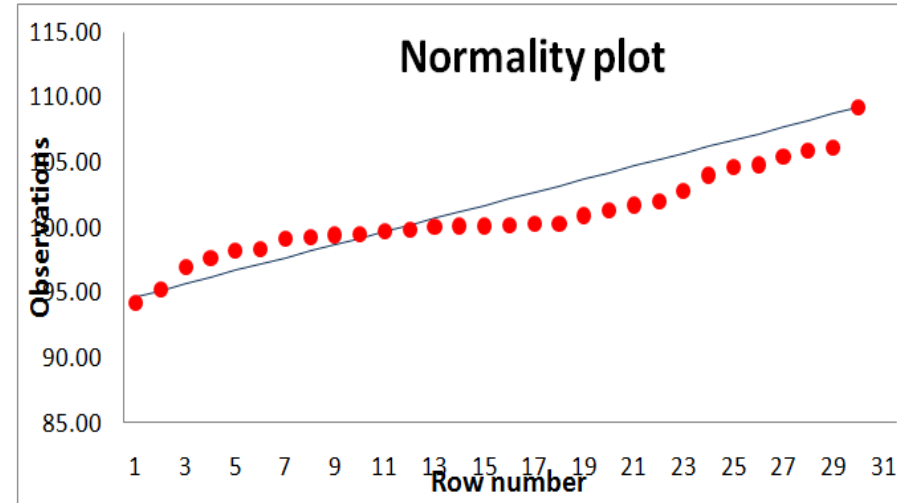

Fig 3 Normality plot of BATCH F1 


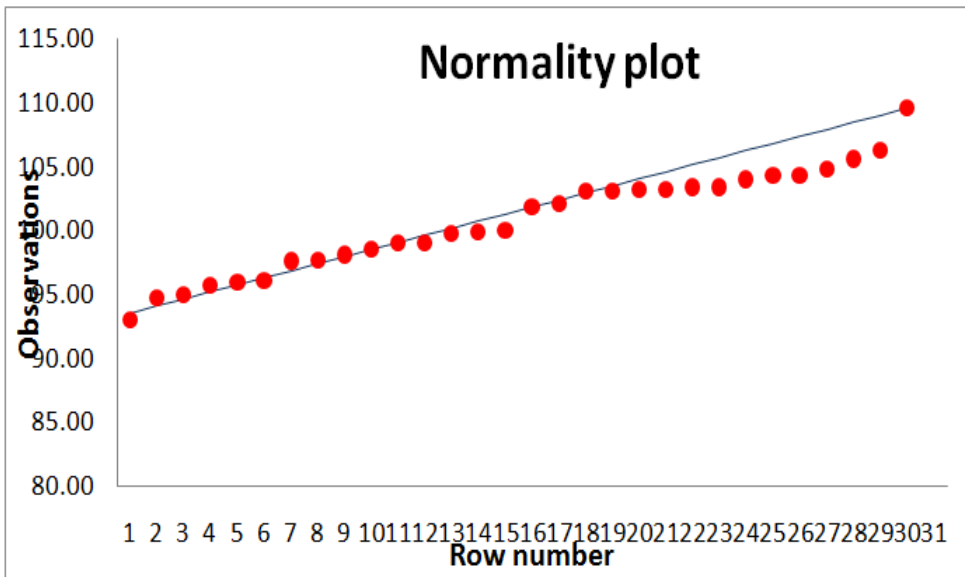

Fig 2. Normality plot of BATCH F2

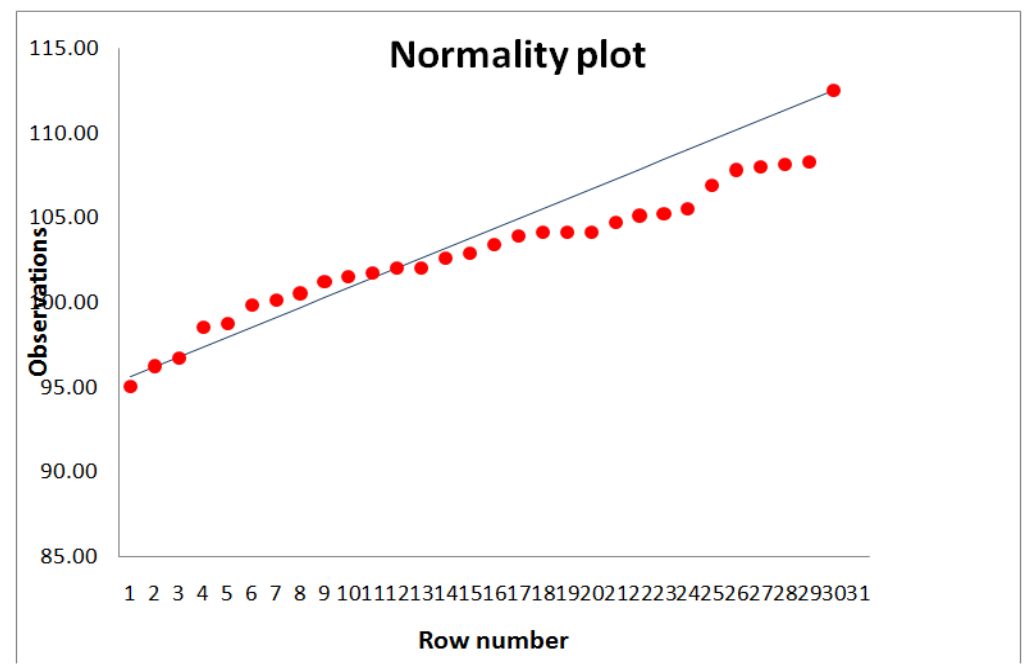

Fig 3. Normality plot of BATCH F3

Table 2. STATISTICAL INFERENCE OF BATCH F1

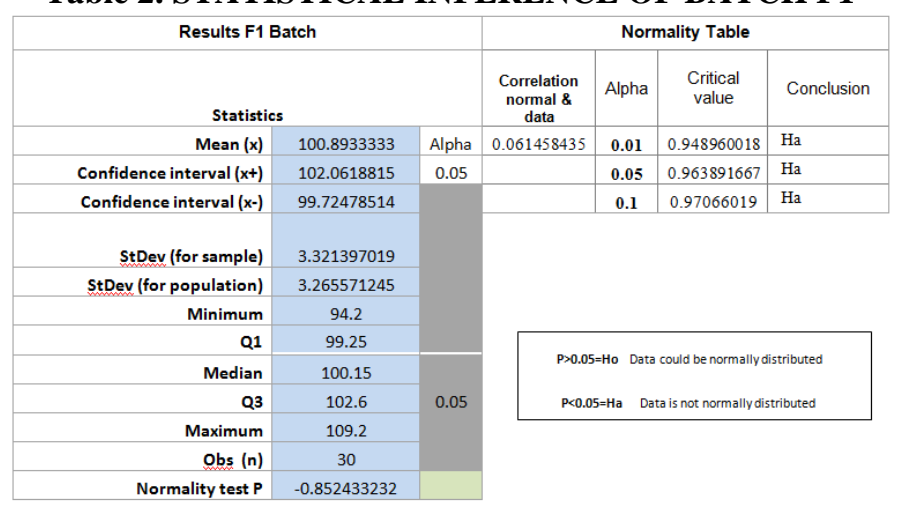

Table 3.STATISTICAL INFERENCE OF BATCH F2 


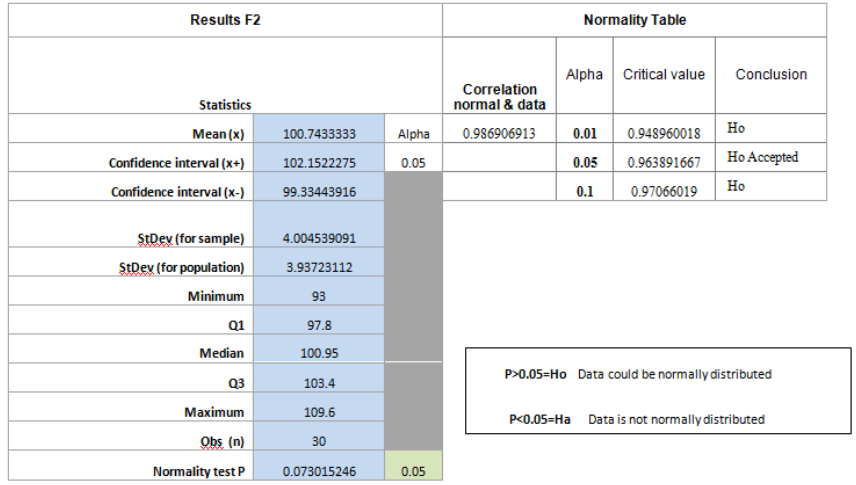

Table 4. STATISTICAL INFERENCE OF BATCH F3

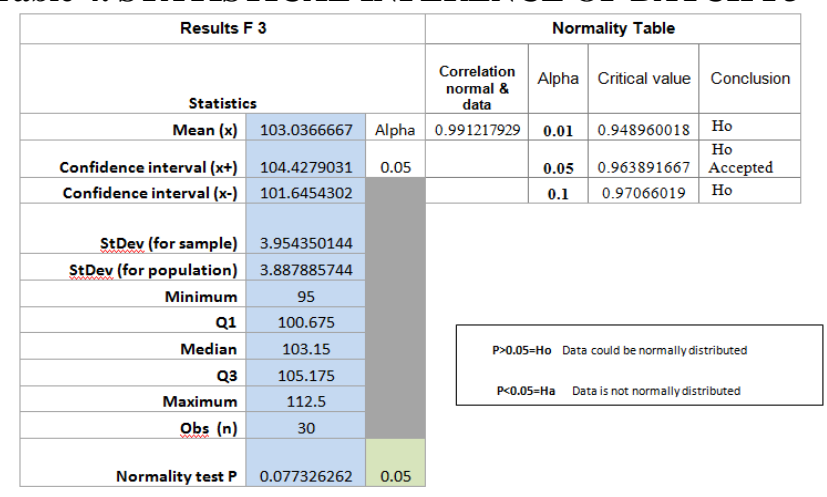

\subsection{Graphic Evaluations As per Histograms}

Graphic evaluations of all batches have computed in graphic prism pad soft ware ${ }^{3}$ Histograms of all batches F1, F2 and F3 are indicated in Fig 6, 7 and 8

Fig 6 Histogram of BATCHF1

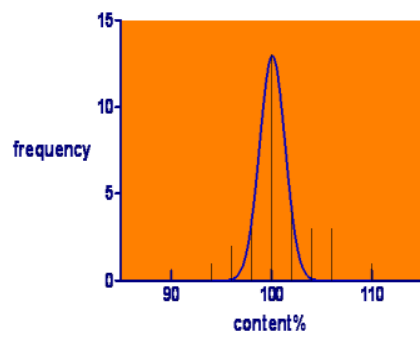

3.3 Box Plots
Fig 7 Histogram of BATCHF2
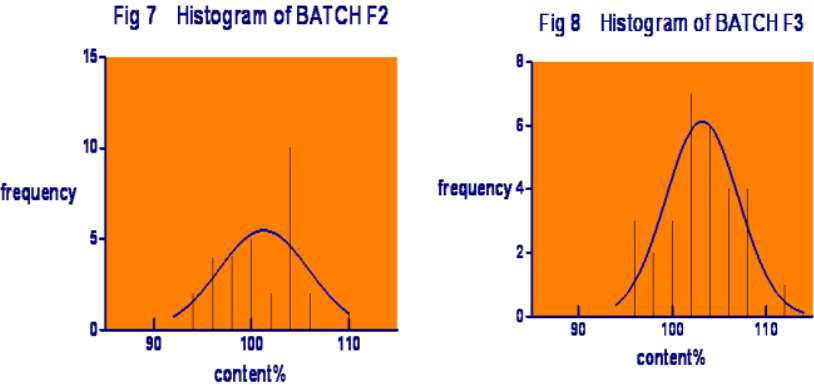

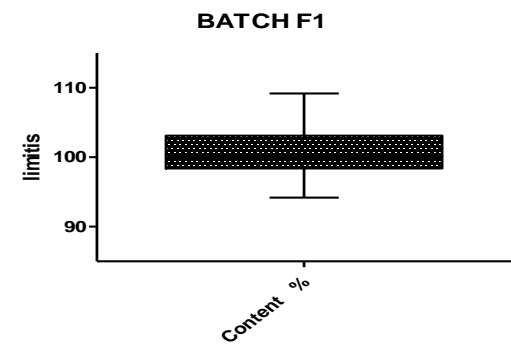

Fig. 9 Box plot of Batch F1 


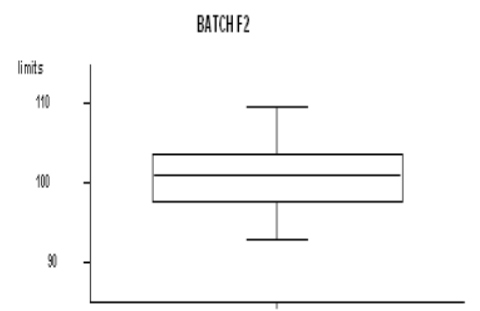

Fig. 9 Box plot of Batch F2

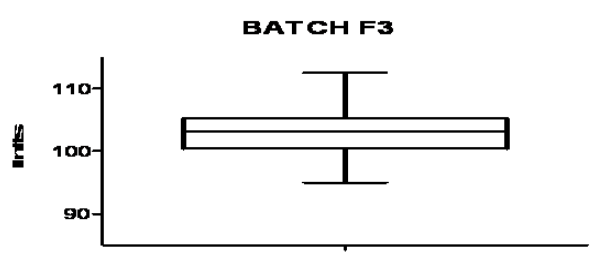

Fig11. Box plot of Batch F2

\subsection{Tolerance intervals}

Tolerance intervals parametric is another approach to find out is statement about the data values M.G Naterella ${ }^{4}$ as well as G.Hunter ${ }^{5}$ et all explained that tolerance interval is a statistical statement about the data value themselves.

For these data $95 \% / 95 \%$ tolerance calculated

The procedure of computing Tolerance intervals parametric are as follows:

1. identify the continuous data

2. Find the sample size $n$

3. Calculate $\bar{X}$

4. Calculate Standard deviations

5. Select $\alpha$, the significance $(1-\alpha)=\gamma=0.95$

6. Select $\mathrm{P}$ the proportion of the population values

7. Find $K=2.549$ for $n$ from Appendix $1^{6}$

8. Calculate $\bar{X} \pm K \mathrm{~s}$

9. We are $100(1-\alpha) \%$ confidence that $\mathrm{P} \%$ of the population values will lies between $\bar{X}-K \mathrm{~s}$ and $\bar{X}+K \mathrm{~s}$

\subsubsection{Results of Tolerance intervals all batches}

Tolerance intervals for batch $F 1$

$\bar{X}+K \mathrm{~s}=\mathbf{1 0 9 . 2 \%}$

$\bar{X}-K s=92.3 \%$

Tolerance intervals for batch $F 2$

$\bar{X}+K \mathrm{~s}=\mathbf{1 1 0 . 8} \%$

$\bar{X}-K s=90.55 \%$

Tolerance intervals for batch $\mathrm{F31}$

$\bar{X}+K \mathrm{~s}=\mathbf{1 1 3 . 4 3} \%$

$\bar{X}-K \mathrm{~s}=93 \%$

As per above estimation we can predict that all tolerances are lies between comfort zone Which have already been fulfill the criteria as set for pharmacopeial limits stated in USP ${ }^{2}$

mentioned in APPENDIX II

\section{5 $\mathbf{C}_{\mathbf{P K}}$ Calculations}

The $\mathrm{C}_{\mathrm{pk}}$ approach is that one would calculate single summary number that indicates the overall capability and high degree of assurance needed for the process. There are various other approaches like 
Bergum's ${ }^{7}$ method and Ruston et all and chou\& Anderson ${ }^{8}$ also emphasized and utilized these calculation with respect to probabilities and operating curve analyses

Formula for $\mathbf{C}_{\mathrm{pk}}$
\[ \mathrm{CPk}=\text { minimum (CPL, CPU) } \]
\[ \boldsymbol{C P \boldsymbol { U }}=\frac{(\boldsymbol{U S} \boldsymbol{L}-\overline{\boldsymbol{X}})}{\mathbf{3 s}} \]
Where:
USL is the upper specification limit
LSL is the lower specification limit
$\overline{\boldsymbol{X}}$ is the average of the sample
$\boldsymbol{s}$ is the standard deviation of the sample
\[ \boldsymbol{s}=\sqrt{\frac{\sum\left(\boldsymbol{x}_{\mathbf{1}}-\boldsymbol{x}\right)^{2}}{\boldsymbol{n}-\mathbf{1}}} \]

\subsubsection{Results of all batches}

\section{$\mathrm{C}_{\mathbf{P k}}$ Result for batch $\mathrm{F} 1$}

$\mathrm{C}_{\mathrm{Pk}}(\min )=$.

$\mathrm{C}_{\mathrm{Pk}}(\max )=$.

$\mathrm{C}_{\mathrm{PK}}$ Result for batch $\mathrm{F} 2$

$\mathrm{C}_{\mathrm{Pk}}(\min )=$.

$\mathrm{C}_{\mathrm{Pk}}(\max )=$.

$\mathrm{C}_{\mathrm{PK}}$ Result for batch $\mathrm{F} 3$

$\mathrm{C}_{\mathrm{Pk}}(\min )=$.

$\mathrm{C}_{\mathrm{Pk}}(\max )=$.

\section{Result \& Discussion}

The data form a single group with the majority close to $100 \%$. The values range from $\sim 94-110 \%$.in in batch $\mathrm{F} 1$

We can conclude that the data are centered near the target and lie within the specifications. To further study the shape of the data, we can compare it to the ideal shape, the normal distribution. The other two batches F2 and F3 are also confirmed with slight difference of patterns. Results of normality plots and their inferences have been reported in table 10.11 and 12 . These profiles are provide notable results that data could be normally distributed

\section{Histogram analysis}

The data were collected throughout the production run and are representative of the process over time. To evaluate this, the 30 data values were plotted in Figure 4 as a time plot to look for any obvious trends or evidence of non randomness. This non randomness can be calculated and predicted in the Histogram analysis. The comprehensive results and Histograms of all three batches mentioned in Figure 6,7 and 8 as per these graphic illustration along with results. We concluded that the data didn't show an upward or downward trend or other obvious nonrandom patterns.

\section{Box plot analysis}

The batches were also compared graphically with a box plot as shown in figures 9,10 and 11

The center line of the box plot is the median or middle value, of the data. The top of box is the $75 \%$ point and the bottom of the box is the $25 \%$. These graphs mentioned in figures 9,10 and 11

Thus, the box plot gives a visual comparison of the center line of the data sets as well as a comparison of variability. Formal statistical tests also determined whether it is appropriate to combine the data from these batches

\section{Tolerance intervals analysis}

Results of content uniformity with respect to Tolerance intervals also very significant for example For these data, the $95 \% / 95 \%$ tolerance interval for batch F1 is 92.3 to 109.2 , which is interpreted as , "We are $95 \%$ 
confident that $95 \%$ of all of the data values from these process will lie in interval 92.3 to 109.2." because the tolerance interval is smaller than the specification of $85-115 \%$, we can be quite confident that this process will be able to meet this specification if it continues to perform in the same manner, assuming the data are approximately normally distributed The other two batches F1, F2 and F3 also have almost same statistical meaning as per above description

\section{Process capabilities analysis}

A comprehensive analyses of all three batches also proceeded with respect to process capability indices

Table 5.Summary of all batches

\begin{tabular}{|lrrrrc|}
\hline Batch & $\mathrm{n}$ & \multicolumn{1}{c}{ Mean } & $\mathrm{SD}$ & $\mathrm{C}_{\mathrm{pk}}$ & Tolerance interval \\
\hline F1 & 30 & 100.8 & 3.32 & 1.42 & $92.3-109.2$ \\
F2 & 30 & 100.7 & 4 & 1.19 & $90.55-110.8$ \\
F3 & 30 & 103.03 & 3.95 & 1.01 & $93-113.43$ \\
All & 90 & 101.5 & 3.75 & 1.206 & \\
\hline Specification= 85-115\% & \multicolumn{5}{l}{} \\
\hline
\end{tabular}

Table 5 is provide sufficient evidence that over all $\mathrm{C}_{\mathrm{pk}}$ of all batches computed as 1.206 in contrast to individual of batch F1, F2 and F3 usually if $\mathrm{C}_{\mathrm{pk}}$ is greater than 1.33 this value indicate that process capable and center focused. The batch F1 is fulfill the this specific requirements while other two batches going towards this value therefore, we can predict that process has tendency to achieve value as per idealized process capability assumptions. While Average $\mathrm{C}_{\mathrm{pk}}=1.206$ is satisfactory agreement of overall process of three batches

\section{Acceptable $\mathbf{C}_{\mathrm{pk}}$}

Given the $\mathrm{C}_{\mathrm{pk}}$ values from the three batches and the overall $\mathrm{C}_{\mathrm{pk}}$, the question now arises, what is an acceptable $\mathrm{C}_{\mathrm{pk}}$ ? The value of $\mathrm{C}_{\mathrm{pk}}$ is related to the probability that the units of a product will be outside of the specifications. As shown in Table 5 The larger $\mathrm{C}_{\mathrm{pk}}$ value, the lower the number of units, or percentage that will be of specifications assuming a normal distribution. In establishing an appropriate process capability, $\mathrm{C}_{\mathrm{pk}}$ for a quality characteristic, one must perform a risk-benefit analysis from the user's point of view.

Acceptable $C_{p k}$ values generally range from 1 to 2 for the quality characteristic of pharmaceuticals products $\mathrm{C}_{\mathrm{pk}}<1$ are economical even for no risk

\section{Conclusion}

FDA's definition of validation was published in May 1987, but there has been no commonly accepted measure of the required "high degree assurance." This project has presented two statistical techniques, the tolerance interval and $\mathrm{Cpk}$, as appropriate and simple measures of the degree of assurance needed for validation. The current study of tablets from an actual validation illustrates the approach. The three batches were very similar normality plots , histogram, statistical inference, confidence intervals were combined to get an overall process capability, Cpk, of 1.206 , versus the 1.33 desired. The process is going towards capability and will meet its content uniformity specification with a high degree of assurance. These recommendations also confirmed by rushton and chou ${ }^{9,10}$ by operating curves

Along with above finding we can also be explain process validation by quality tools which give better under standing of process. This project has also great importance for pharmaceutical industry exclusively for those industry. Which are struggling to stream line their process by validation and improvements by proper application.

\section{References:}

[1] Kirffer validation, risk, benefit analysis PDA Journal pharmaceutical. Science \& Technology 49 (5) 249 - 252 sep 1995

[2] Robert A .Nash, Alferd H. Watcher Pharmaceutical process validation $3^{\text {rd }}$ edition Volume 129

[3] Graph prism pad Version 5

[4] M.G Natrella Experimental statistics (Hand book 91. US Government printing office ,Washington DC. 1963

[5] Box, G. E. P., Gunter, W. G., and Hunter, J. S. Statistics for Experimenters: An Introduction to Design, Data Analysis, and Model Building. New York: Wiley (1978)

[6]. Douglus C. Montgomery Introduction to statistical Quality Control $6^{\text {th }}$ edition

[7] J.Bergum Constructing Accepting limits for multiple stage Tests Drug Dev.Ind.Pharm 16 (14) 2153-2166 1990

[8] SubirGhosh,William R. Schucany, William BR Smith Statistics of Quality Volume 153

[9] Chuo .Y addition to the table of normal integral. Communications in statistics B10(5)537-538

[10] Rushton, S.( 1950) On a sequential t test Biometrica 37:326-333 